\title{
A COUNTEREXAMPLE ON THE SEMICONTINUITY OF MINIMA
}

\author{
FERNANDO LUQUE-VÁSQUEZ AND ONÉSIMO HERNÁNDEZ-LERMA
}

(Communicated by Joseph S. B. Mitchell)

\begin{abstract}
Let $X$ and $Y$ be metric spaces, $\Phi$ a multifunction from $X$ to $Y$, and $v$ a real-valued function on $X \times Y$. We give an example in which $\Phi$ is continuous, and $v$ is continuous, inf-compact and bounded below, but the minimum function $v^{*}(x):=\inf _{y \in \Phi(x)} v(x, y)$ on $X$ is not lower semicontinuous.
\end{abstract}

\section{INTRODUCTION}

An important problem in optimization, control theory and related fields is the following. Let $X$ and $Y$ be metric spaces, and $\Phi$ a multifunction (or setvalued map) from $X$ to $Y$ with $\Phi(x) \neq \phi$ for all $x$ in $X$. Given a real-valued function $v$ on the graph $\operatorname{Gr}(\Phi)$ of $\Phi$, which is defined as

$$
\operatorname{Gr}(\Phi):=\{(x, y) \mid x \in X, y \in \Phi(x)\}
$$

the problem is to give conditions under which the minimum function

$$
v^{*}(x):=\inf _{y \in \Phi(x)} v(x, y), \quad x \in X,
$$

is lower semicontinuous (1.s.c.).

For example, a well-known result (see, e.g., [1], p. 14, Proposition 1.7; [3], p. 148, Proposition 7.32) states that $v^{*}$ is l.s.c. if $\Phi(\cdot) \equiv Y$ is compact and $v$ is l.s.c. Other known results include Theorems 1 and 2 below.

Theorem 1 (cf. [2], p. 116, Theorem 2; [5], Theorem 10.2). Suppose that $\Phi$ is compact-valued and upper semicontinuous. If $v$ is l.s.c. and bounded below, then so is $v^{*}$.

Theorem 2 (cf. [4], Lemma 3.2(f)). Suppose that $\operatorname{Gr}(\Phi)$ is a Borel subset of $X \times Y$, and that $v$ is l.s.c. and inf-compact on $\operatorname{Gr}(\Phi)$-where inf-compactness means that for every real number $r$ and $x$ in $X$, the set $\{y \in \Phi(x) \mid v(x, y) \leq r\}$

Received by the editors March 2, 1994.

1991 Mathematics Subject Classification. Primary 90C48; Secondary 26E25.

Key words and phrases. Minimization problem, multifunctions, measurable selections.

Research partially supported by the Consejo Nacional de Ciencia y Tecnologia (CONACyT) grant \#1132-E9206. 
is compact. If, in addition, the multifunction

$$
x \mapsto \Phi^{*}(x):=\left\{y \in \Phi(x) \mid v^{*}(x)=v(x, y)\right\}
$$

is l.s.c., then $v^{*}$ is l.s.c.

The essential difference between Theorem 1 and Theorem 2 is that the compactness and upper semicontinuity of $\Phi$ in the former is replaced by infcompactness of $v$ and lower semicontinuity of $\Phi^{*}$ in the latter. We thank the referee for pointing out that Theorem 1 also holds even if the boundedness assumption on $v$ is dropped, which can be deduced, for instance, from Theorem 1.4.16 in J.-P. Aubin and H. Frankowska, Set-valued analysis, Birkhäuser, 1990.

The following example shows that $v^{*}$ fails to be l.s.c. if the assumptions in the above theorems are weakened.

\section{THE EXAMPLE}

Suppose that $X=\mathbb{R}$ and $Y=\Phi(x)=[0, \infty)$ for all $x \in X$, and let $v$ be defined as

$$
\begin{aligned}
v(x, y) & :=1+y & & \text { if }(x \leq 0) \text { or }(x>0,0 \leq y \leq 1 / 2 x), \\
& :=(2+1 / x)-(2 x+1) y & & \text { if } x>0,1 / 2 x<y \leq 1 / x, \\
& :=y-1 / x & & \text { if } x>0, y>1 / x .
\end{aligned}
$$

Then the multifunction $x \mapsto \Phi(x)$ is continuous, and $v$ is continuous, infcompact on $\operatorname{Gr}(\Phi)=\mathbb{R} \times[0, \infty)$ and bounded below (nonnegative), but

$$
v^{*}(x)= \begin{cases}1 & \text { if } x \leq 0 \\ 0 & \text { if } x>0\end{cases}
$$

is not l.s.c.

\section{REFERENCES}

1. J.-P. Aubin, Optima and equilibria, Springer-Verlag, Berlin, 1993.

2. C. Berge, Topological spaces, Macmillan, New York, 1963.

3. D. P. Bertsekas and S. E. Shreve, Stochastic optimal control: The discrete time case, Academic Press, New York, 1978.

4. O. Hernández-Lerma and W. J. Runggaldier, Monotone approximations for convex stochastic control problems, J. Math. Syst. Estimation and Control 4 (1994), 99-140.

5. M. Schäl, Conditions for optimality and for the limit of n-stage optimal policies to be optimal, Z. Wahrsch. Verw. Gebiete 32 (1975), 179-196.

Departamento de Matemáticas, Universidad de Sonora, Rosales y BouleVard Luis EnCINAS, 83000 Hermosillo, SONORA, Mexico

Departamento de Matemáticas, CinVeSTaV-ipN, A. Postal 14-740, 07000 México, D.

F., MEXICo

E-mail address: ohernand@math.cinvestav.mx 